\title{
Transient Receptor Potential Ankyrin-1 and Vanilloid-3 Differentially Regulate Endoplasmic Reticulum Stress and Cytotoxicity in Human Lung Epithelial Cells After Pneumotoxic Wood Smoke Particle Exposure $\mathrm{g}$
}

\author{
Nam D. Nguyen, Tosifa A. Memon, Katherine L. Burrell, Marysol Almestica-Roberts, \\ Emmanuel Rapp, Lili Sun, Abigail F. Scott, Joseph E. Rower, Cassandra E. Deering-Rice, \\ and Christopher A. Reilly
}

Department of Pharmacology and Toxicology, Center for Human Toxicology, University of Utah, Salt Lake City, Utah

Received April 24, 2020; accepted August 27, 2020

\section{ABSTRACT}

This study investigated the roles of transient receptor potential (TRP) ankyrin-1 (TRPA1) and TRP vanilloid-3 (TRPV3) in regulating endoplasmic reticulum stress (ERS) and cytotoxicity in human bronchial epithelial cells (HBECs) treated with pneumotoxic wood smoke particulate matter (WSPM) and chemical agonists of each channel. Functions of TRPA1 and TRPV3 in pulmonary epithelial cells remain largely undefined. This study shows that TRPA1 activity localizes to the plasma membrane and endoplasmic reticulum (ER) of cells, whereas TRPV3 resides primarily in the ER. Additionally, treatment of cells using moderately cytotoxic concentrations of pine WSPM, carvacrol, and other TRPA1 agonists caused ERS as a function of both TRPA1 and TRPV3 activities. Specifically, ERS and cytotoxicity were attenuated by TRPA1 inhibition, whereas inhibiting TRPV3 exacerbated ERS and cytotoxicity. Interestingly, after treatment with pine WSPM, TRPA1 transcription was suppressed, whereas TRPV3 was increased. TRPV3 overexpression in HBECs conferred resistance to ERS and an attenuation of ERSassociated cell cycle arrest caused by WSPM and multiple prototypical ERS-inducing agents. Alternatively, short hairpin RNA-mediated knockdown of TRPV3, like the TRPV3 antagonist, exacerbated ERS. This study reveals previously undocumented roles for TRPA1 in promoting pathologic ERS and cytotoxicity elicited by pneumotoxic WSPM and TRPA1 agonists, and a unique role for TRPV3 in fettering pathologic facets of the integrated ERS response.

\section{SIGNIFICANCE STATEMENT}

These findings provide new insights into how wood smoke particulate matter and other transient receptor potential ankyrin1 (TRPA1) and transient receptor potential vanilloid-3 (TRPV3) agonists can affect human bronchial epithelial cells and highlight novel physiological and pathophysiological roles for TRPA1 and TRPV3 in these cells.

\section{Introduction}

Wood/biomass smoke and particulate emissions [wood smoke particulate matter (WSPM)] are a major human health risk. Exposure to high concentrations of particulate material (PM) from multiple types of wood and biomass has been linked to increased rates of hospital admissions for respiratory complications due to acute lung inflammation, tissue damage, and respiratory distress (Swiston et al., 2008; Ghio et al., 2012;

This work was supported by National Institutes of Health National Institute of Environmental Health Sciences [Grants ES017431 and ES027015]. N.D.N. was supported in part by a University of Utah Undergraduate Research Opportunities award, and M.A.-R. was supported by a National Institutes of Health National Institute of General Medical Sciences Diversity Supplement award associated with [Grant GM121648]. K.L.B. received partial support from a University of Utah Associated Regional and University Pathologists, Inc. fellowship. Imaging was performed at the Florescence Microscopy Core Facility, a part of the Health Sciences Cores at the University of Utah. Microscopy equipment was obtained using a National Center for Research Resources Shared Equipment Grant [RR024761]. The University of Utah Flow Cytometry Facility is supported by National Institutes of Health National Cancer Institute [Grant CA042014].

https://doi.org/10.1124/molpharm.120.000047.

S This article has supplemental material available at molpharm. aspetjournals.org.
Reid et al., 2016; Liu et al., 2017). Long-term exposure is also associated with the development and exacerbation of chronic diseases including asthma, emphysema, and chronic obstructive pulmonary disease (Laumbach and Kipen, 2012; Olloquequi and Silva O, 2016). Currently, mechanisms by which WSPM affect the respiratory tract are not fully understood.

WSPM is a complex mixture of solids and condensed chemicals with variable pneumotoxic potential (Kim et al., 2018). Our laboratory has shown that WSPM from pine, mesquite, and other fuels differentially activate the transient receptor potential ankyrin-1 (TRPA1) and transient receptor potential vanilloid-3 (TRPV3) ion channels (Shapiro et al., 2013; Deering-Rice et al., 2018). Neuronal TRPA1 is a mediator of pulmonary irritation and inflammation (Andrè et al., 2008; Bessac et al., 2008), but the functions of TRPA1 in nonneuronal cells are less understood. Studies have shown that pine and other forms of WSPM, pure TRPA1 agonists [i.e., allyl isothiocyanate (AITC) and coniferaldehyde], as well as diesel exhaust particles, stimulate the expression and secretion of gel-forming mucins (MUC5AC and 5B), as well 
as MUC4, by activating TRPA1 in primary human bronchial epithelial cells (HBECs) (Deering-Rice et al., 2019; Memon et al., 2020). Consequences of TRPV3 activation in HBECs by WSPM have also been explored (Deering-Rice et al., 2018). For example, TRPV3 overexpression in BEAS-2B lung cells stably overexpressing TRPV3 (TRPV3OE cells) was found to increase the cytotoxicity of pine WSPM, and TRPV3 inhibition reduced the cytotoxicity. Additionally, a TRPV3 antagonist prevented changes in pulmonary function associated with subchronic pine WSPM treatment in mice. However, detailed mechanisms for how TRPA1 and TRPV3 ultimately influenced the cytotoxicity of WSPM were not defined, leaving critical knowledge gaps surrounding the physiologic and pathophysiological functions of these channels in airway epithelial cells.

Our laboratory previously demonstrated that activation of TRPV1 in HBEC cells using cell-permeable agonists caused lung epithelial cell death through disruption of endoplasmic reticulum (ER) calcium homeostasis and initiation of pathologic ER stress (ERS) (Thomas et al., 2011, 2012). ERS is a conserved collection of processes that become activated when protein folding and/or secretion are disrupted (Korfei et al., 2016), as outlined by Scheme 1. ERS has been linked to various lung pathologies including mucus hypersecretion in individuals with asthma and cystic fibrosis, due to ectopic inositol requiring element-1 (IRE1)- $\beta$ activity (Martino et al., 2013). IRE1 is a key regulatory element of the integrated ERS response and is generally considered to be central in the coordination of adaptive responses during stress. Additionally, a role for the ERS-regulated gene DNA damage inducible transcript-3 (DDIT3) has been shown in idiopathic pulmonary fibrosis (Yao et al., 2016), and a role for ERS was shown in a model of cigarette smoke-induced pulmonary inflammation and emphysema (Wang et al., 2017).

An array of stimuli can induce ERS and include oxidative stress, misfolding of proteins due to mutations or chemical modification, viral infection, glucose starvation, as well as calcium store depletion, including through the activation of transient receptor potential (TRP) channels (Xu et al., 2005; Thomas et al., 2012). Although several studies have shown that ERS can also occur in HBECs and in lungs exposed to PM (Watterson et al., 2009; Xu et al., 2019), a role of TRPA1 in regulating ERS and the roles of TRPA1 and ERS in WSPM toxicity have not been explored. It is also currently unknown whether TRPV3 can regulate ERS and/or cytotoxicity in HBECs as a result of stimulation by WSPM or other toxins.

The hypothesis of this study was that pine WSPM, as well as TRPA1 and TRPV3 agonists, would promote HBEC cytotoxicity through activation of TRPA1 and/or TRPV3, ER calcium depletion and initiation of pathologic ERS. The objectives of this study were 1) to delineate mechanisms by which pneumotoxic WSPM affect HBEC viability and 2) to elucidate roles for

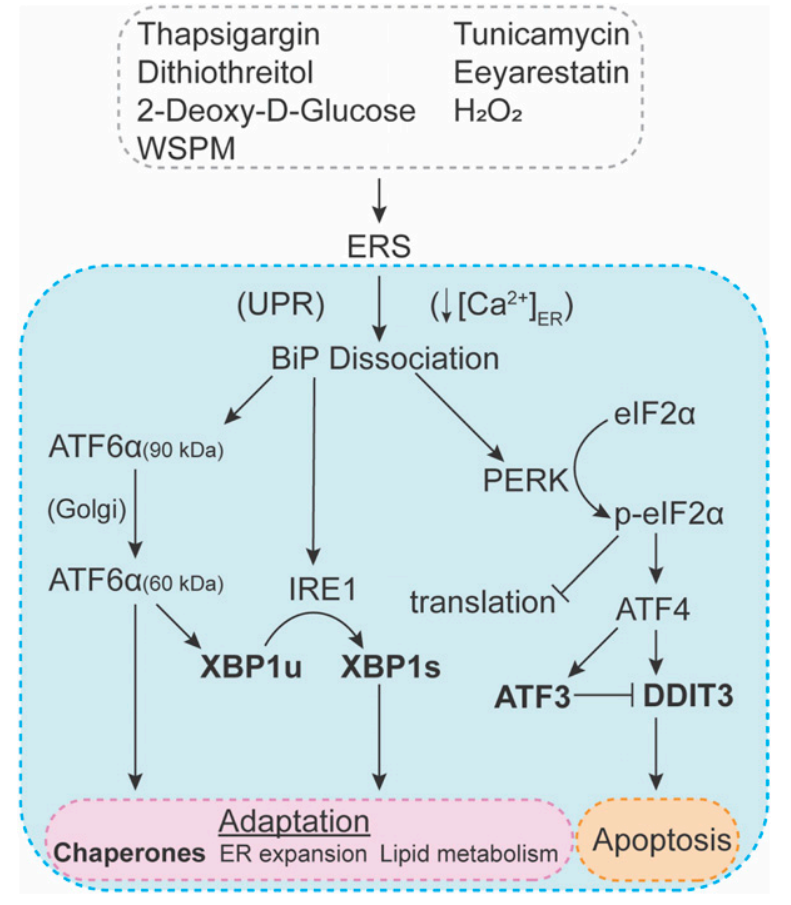

Scheme 1. Scheme outlining the integrated ERS response, common stimuli, and common cellular responses. The overall ERS pathway is highlighted in blue, with bold text specifying the biomarkers that were used as indices of ERS in this study. The various ERS-inducing agents that were used are listed in the gray box. BiP, binding immunoglobulin protein; eIF2, eukaryotic initiation factor 2; p-eIF2, phosphorylated eukaryotic initiation factor 2; UPR, unfolded protein response.

TRPA1 and TRPV3 in regulating cytotoxicity caused by a model pneumotoxic pollutant, pine WSPM, and prototypical TRPA1 and TRPV3 agonists that mimic the effects of WSPM on these channels.

\section{Materials and Methods}

Chemicals. All chemicals were purchased from Cayman Chemical or Sigma-Aldrich unless otherwise specified. A TRPV3 antagonist previously described by Hydra Biosciences, 2-(5-trifluoromethyl-pyridine-2-ylsulfanyl)-1-(8-methyl-3,4-dihydro-2H-quinolin-1-yl)-ethanone, was synthesized as previously described (Deering-Rice et al., 2014). For simplicity and consistency with our prior studies, we refer to this molecule as simply "TRPV3 antagonist."

Size-fractionated pine WSPM and other forms of WSPM (i.e., juniper, apple wood, range grass, Colorado sage, goat, sheep, and cow dung smoke PM) were prepared using a laboratory furnace as previously described (Shapiro et al., 2013). Juniper and Colorado sage were collected in the fall season near the four corners region of Colorado. Apple wood was from tree trimmings of a mature tree grown in Salt Lake City, UT, and range grass was collected from the Salt Lake City foothills, also in the fall. Animal dung was collected fresh in

\footnotetext{
ABBREVIATIONS: AITC, allyl isothiocyanate; ATCC, American Type Culture Collection; ATF, activating transcription factor; DDIT3, DNA damage inducible transcript-3; ER, endoplasmic reticulum; ERS, endoplasmic reticulum stress; HBEC, human bronchial epithelial cell; HBEC3-KT cells, normal human bronchial epithelial cells immortalized with cyclin-dependent kinase 4 and human telomerase reverse transcriptase; HEK, human embryonic kidney; HSPA1A, heat shock 70 kDa protein 1; IRE1, inositol requiring element-1; LHC-9, Lechner and LaVeck medium; $\beta 2 M$, beta-2 microglobulin; PERK/elF2 $\alpha \mathrm{K} 3$, protein kinase R-like endoplasmic reticulum kinase; PM, particulate material; qPCR, quantitative real-time polymerase chain reaction; SAEC, small airway epithelial cell; shRNA, short hairpin RNA; TRP, transient receptor potential; TRPA1, transient receptor potential ankyrin-1; TRPV3, transient receptor potential vanilloid-3; TRPV3 antagonist, 2-(5-trifluoromethyl-pyridine-2-ylsulfanyl)-1-(8methyl-3,4-dihydro-2H-quinolin-1-yl)-ethanone; TRPV3OE cells, BEAS-2B cells stably overexpressing TRPV3; WSPM, wood smoke particulate matter; XBP1, X-box binding protein-1; XBP1s, spliced transcript of X-box binding protein-1; XBP1u, unspliced transcript of X-box binding protein-1.
} 


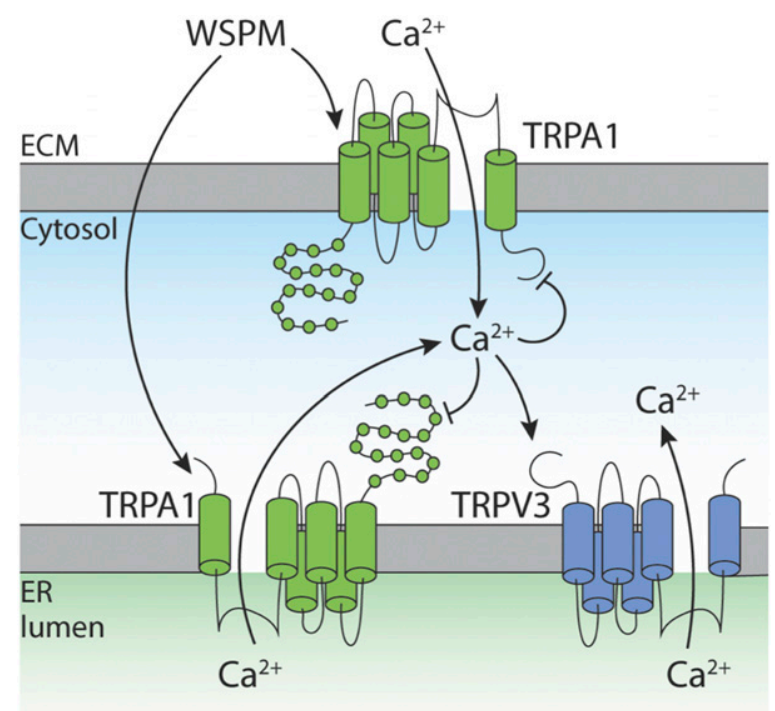

Scheme 2. Proposed mechanism for the regulation of ERS by TRPA1 and TRPV3 in HBECs. WSPM activates TRPA1 and TRPV3 to cause calcium influx and ER calcium release. High intracellular calcium may sensitize/ activate TRPV3 on the ER to promote ER calcium release to drive robust ERS responses. Conversely, high levels of intracellular calcium will desensitize TRPA1, leading to a decrease in ER calcium release and ERS, which appears to be driven by TRPV3 activity. ECM, extracellular matrix.

a clean room facility from animals housed at the US Department of Agriculture Agricultural Research Services facility (Logan, UT), from animals maintained on standard diets. The feces samples were dried at room temperature in a fume hood over multiple days. For all experiments, with the exception of results in Supplemental Figures 1 and 4 , fraction $7(0.43-0.65 \mu \mathrm{m})$ WSPM was used. In all cases, WSPM was solubilized in DMSO at a stock concentration of $115 \mathrm{mg} / \mathrm{ml}$ and diluted into media as needed, with the DMSO content at $2 \%$ or less. Because WSPM is unstable when stored and after resuspension for experiments, materials were not used beyond 3 months after production date and were prepared fresh for each experiment. Furthermore, each batch of WSPM was verified for potency in TRPA1 and TRPV3 calcium flux assays and must fall within a consistent range that we have determined empirically to be an average and to produce a consistent range of results in our various endpoint assays.

Cell Culture. Cells were maintained at $37^{\circ} \mathrm{C}$ and $5 \% \mathrm{CO}_{2}$ in a humidified incubator. TRP channel-overexpressing human embryonic kidney (HEK)-293 cells were generated as previously described and cultured in Dulbecco's modified Eagle's medium-F12 medium supplemented with $5 \%$ fetal bovine serum, $1 \times$ penicillin/streptomycin, and $300 \mu \mathrm{g} / \mathrm{ml}$ geneticin (Deering-Rice et al., 2012). BEAS-2B HBECs [American Type Culture Collection (ATCC), Manassas, VA] were cultured in Lechner and LaVeck (LHC-9) medium (Life Technologies, Carlsbad, CA), and TRPV3OE cells were selected and cultured as previously described (Deering-Rice et al., 2018). Primary human lobar bronchial epithelial cells (lobar HBECs; donor identifier: 01344) and human small airway epithelial cells (SAECs; donor identifier: 00656) were purchased from Lifeline Cell Technology (Frederick, MD). Normal human bronchial epithelial cells immortalized with cyclindependent kinase 4 and human telomerase reverse transcriptase (HBEC3-KT cells) were purchased from ATCC. HBEC and SAECs were cultured using the BronchiaLife epithelial airway medium complete kit from Lifeline Cell Technology. HBEC3-KT cells were cultured with airway epithelial cell basal medium supplemented with the bronchial epithelial cell growth kit from ATCC. The vehicle used for mRNA expression, Western blot, cytotoxicity, and flow cytometry studies was fresh medium, either fresh LHC-9 for BEAS-2B cells or
BronchiaLife epithelial airway medium for lobar HBECs. Note: The lobar HBECs, SAECs, and HBEC3-KT cells used in this study all have the TRPV1 I585I/V (rs8065080) genotype, which has previously been shown to correlate with higher levels of TRPA1 expression (DeeringRice et al., 2016). Alternatively, the BEAS-2B cells and derivative lines thereof have the I585I/I genotype and consequently lower levels of TRPA1 expression. All of the cells used in this study were also homozygous wild-type for the TRPA1 R3C and R58T polymorphisms, rs13268757 and rs16937976, respectively, which have also been associated with a gain of function for some particle agonists and increased risk for poor asthma control (Deering-Rice et al., 2015). As such, the TRPV1 and TRA1 genotypes should be considered as a variable in future studies of TRPA1 agonists on HBECs because the genotype could affect how the cells respond to various forms of PM and TRPA1 agonists. Genotyping of the cells was performed as previously described (Deering-Rice et al., 2015, 2016). Finally, it was observed that the expression of TRPA1 decreased in primary cells with passaging. As such, to minimize experimental variability due to changes in basal levels of TRPA1 and TRPV3 expression, the primary HBECs were used for a maximum of five passages after receipt from the supplier.

Calcium Flux Assays. Pine WSPM- and TRP agonist-induced calcium flux was measured using the Fluo-4 Direct assay kit (Invitrogen, Carlsbad, CA). Calcium flux was imaged on an EVOS FL Auto microscope (ThermoFisher, Waltham, MA) as previously described (Deering-Rice et al., 2011, 2012). Briefly, cells were imaged at $10 \times$ magnification using a green fluorescent protein filter set. Cells were maintained at room temperature $\left(\sim 22-23^{\circ} \mathrm{C}\right)$, and agonist treatments were added to cells at $3 \times$ concentration in LHC-9, which contains $111.1 \mu \mathrm{M}$ calcium. Images were captured every 20 seconds for 100 seconds. Changes in fluorescence were determined using an Image-J-based program, and the values were corrected by subtracting the baseline fluorescence and normalized to the maximum fluorescence intensity elicited by ionomycin $(10 \mu \mathrm{M})$, applied after agonist treatment. In calcium flux experiments where thapsigargin $(2.5 \mu \mathrm{M})$ was used as a pretreatment to deplete ER calcium stores, a transient increase in cealcium occurred. Accordingly, agonists were applied only after the intensity returned to baseline, approximately 5 minutes after thapsigargin application.

Cytotoxicity Assays. For cytotoxicity assays, lobar HBEC cells were cultured in a 96 -well plate at a density of 25,000 cells per well for 24 hours. After 24 hours, cells were then treated with pine WSPM suspended in culture media at increasing concentrations for an additional 24 hours. When the effects of TRPA1 and TRPV3 antagonists were tested, pine PM was applied at a fixed concentration of $20 \mu \mathrm{g} / \mathrm{cm}^{2}$. After treatment, residual viability was measured using the Dojindo Cell Counting Kit-8 (Dojindo, Rockville, MD) according to the manufacturer's protocol.

Immunocytochemical Localization of TRPV3 in HBECs. Lobar HBECs were grown on either ethanol and flame-sterilized coverslips or eight-well chamber slides, which were coated with LHC basal medium fortified with collagen ( $30 \mu \mathrm{g} / \mathrm{ml})$, fibronectin $(10 \mu \mathrm{g} / \mathrm{ml})$, and bovine serum albumin fraction $\mathrm{V}(100 \mu \mathrm{g} / \mathrm{ml})$. Cells were then fixed with $4 \%$ paraformaldehyde and permeabilized with $0.2 \%$ Triton $\mathrm{X}-100$. Nonspecific antibody binding was blocked by incubating cells in $10 \%$ normal goat serum for 1 hour at room temperature $\left(\sim 22^{\circ} \mathrm{C}\right)$. Cells were then incubated with a mouse monoclonal primary antibody for TRPV3 (1:200, 73-043; Neuromab, Davis, CA) and a rabbit polyclonal primary antibody for the ER biomarker calnexin (1:1000, ab22595; Abcam, Cambridge, MA) overnight at $4^{\circ} \mathrm{C}$, followed by incubation with a goat-anti-mouse secondary antibody conjugated with AlexaFluor488 (1:1000, A-11001; Invitrogen) and a goat-anti-rabbit secondary antibody conjugated with AlexaFluor594 (1:1000, A-11012; Invitrogen). After incubation with the antibodies, cell nuclei were stained with Hoechst 33342, and cells were postfixed with $4 \%$ paraformaldehyde. Coverslips were then mounted to microscope slides with Prolong Gold Antifade Mounting Medium (Invitrogen). Cells were imaged on a Nikon A1R confocal microscope with a $60 \times$ oil 
immersion lens. TRPV3 primary antibody specificity was previously determined by Western blot (Deering-Rice et al., 2018). Additionally, use of this antibody for immunocytochemistry was validated by comparing naïve and TRPV3-overexpressing BEAS-2B HBECs, where staining intensity was far greater in the overexpressing cells (Supplemental Fig. 3B).

Quantitative Real-Time Polymerase Chain Reaction Gene Expression Analysis. Cells were grown to confluence in 12-well plates. After treatment, total RNA was isolated using the PureLink RNA Mini Kit (Invitrogen). Total RNA $(2 \mu \mathrm{g})$ cDNA was synthesized using the ABI High Capacity cDNA Synthesis Kit with RNase Inhibitor (Applied Biosystems, Foster City, CA). The cDNA was then subjected to analysis by quantitative real-time polymerase chain reaction (qPCR) using a Life Technologies QuantStudio 6 Flex instrument. Taqman probe-based assays were used for human TRPV3 (Hs00376854_m1), human TRPA1 (Hs00175798_m1), human activating transcription factor-3 (ATF3; Hs00231069_m1), human DDIT3 (Hs00358796_g1), and human heat shock $70 \mathrm{kDa}$ protein 1 (HSPA1A; Hs00359163_s1). Fluorescent probes and primers to distinguish between the spliced (XBP1s) and unspliced (XBP1u) transcripts of $\mathrm{X}$-box binding protein-1 (XBP1) were designed as described elsewhere (Maiuolo et al., 2011). For both XBP1s and XBP1u, the same external primers were used, each at a final concentration of $900 \mathrm{nM}$ (forward primer: 5'-AAT GAA GTG AGG CCA GTG GC-3'; reverse primer: $5^{\prime}$ TGA AGA GTC AAT ACC GCC AGA A-3'). For XBP1s, the internal probe sequence was designed to span the splice junction between exons 4 and 5 (probe sequence: 5 '-TGC TGA GTC CGC AGC AGG TGC $\left.\mathrm{A}-3^{\prime}\right)$, whereas for $\mathrm{XBP} 1 \mathrm{u}$, the internal probe sequence spanned intron 4 (probe sequence: $5^{\prime}$-CAG CAC TCA GAC TAC GTG-3'). XBP1 probes were fluorescein-amitide (FAM)labeled at the $5^{\prime}$-end, Black Hole Quencher-1 (BHQ1)-labeled at the 3'-end, and used at a final concentration of $250 \mathrm{nM}$. Relative gene expression data were normalized against the housekeeping gene $\beta 2$-microglobulin ( $\beta 2 \mathrm{M}$; Hs00984230_m1) using the $\Delta \Delta \mathrm{Ct}$ method. The absolute number of copies of TRPA1 and TRPV3 mRNA in cells was determined using standard curves for TRPA1, TRPV3, and $\beta 2 \mathrm{M}$.

Western Blot Analysis. Cells were grown to confluence in $25 \mathrm{~cm}^{2}$ flasks. After treatment, total protein was harvested on ice using radioimmunoprecipitation buffer, supplemented with $6 \mathrm{M}$ urea, $1 \%$ SDS, and Halt protease inhibitor (Invitrogen). Lysates were sonicated on ice $3 \times$ for 3 seconds and clarified by centrifugation at $13,000 \mathrm{~g}$ for 15 minutes at $4^{\circ} \mathrm{C}$. Protein concentrations were determined using the bicinchoninic acid method (ThermoFisher). Thirty micrograms of protein was loaded into each well of a $4 \%-12 \%$ Bolt BisTris 12-well gel (Invitrogen) and resolved by electrophoresis for 1 hour at $150 \mathrm{~V}$. Protein was transferred to a polyvinylidene fluoride membrane using the iBlot 2 Gel Transfer Device (Life Technologies). After transfer, the membrane was blocked in SuperBlock (Invitrogen) for 1 hour at room temperature. Primary mouse monoclonal antibodies against DDIT3 (2895; Cell Signaling Technology; Danvers, MA; $1: 1000$ in $5 \%$ bovine serum albumin with $0.1 \%$ sodium azide) and $70 \mathrm{kDa}$ heat shock proteins (ab2787; Abcam; 1:1000 in 5\% bovine serum albumin with $0.1 \%$ sodium azide) were incubated at $4^{\circ} \mathrm{C}$ overnight and used in conjunction with horseradish peroxidase-conjugated sheep-anti-mouse secondary antibodies (GE Health Sciences, Marlborough, MA; 1:10,000 in SuperBlock). SuperSignal West Dura Extended Duration Substrate was added to the membrane, and immunostaining was visualized using autoradiography film. Bands were quantified using densitometry on ImageJ, normalized against the vehicle control.

Flow Cytometry/Cell Cycle Analysis. BEAS-2B and TRPV3OE cells were grown to $\sim 50 \%$ confluence, treated for 4 hours, collected by trypsinization, and fixed in 66\% ice-cold ethanol. After fixation, cell nuclei were stained and analyzed using the Propidium Iodide Flow Cytometry Kit (ab139418; Abcam) according to manufacturer specifications. Analysis of samples was performed at the University of Utah Flow Cytometry Core facility using the BD FACS Canto Analyzer. A sublethal dose of pine WSPM $\left(5 \mu \mathrm{g} / \mathrm{cm}^{2}\right)$ was used for cell cycle analysis experiments to minimize cell damage and death while still capturing cell cycle arrest.

Knockdown of TRPV3 in BEAS-2B HBECs. BEAS-2B cells were transfected with $1 \mu \mathrm{g}$ TRPV3-specific construct D or control/ scrambled short hairpin RNA (shRNA) plasmid DNA provided with the TRPV3 Human shRNA Plasmid Kit (Locus identifier: 162514, TF300805; OriGene Technologies, Rockville, MD) using FUGENE 6 transfection reagent (3:1 reagent to DNA). Stable overexpression of the shRNA construct was selected using resistance to puromycin $(1 \mu \mathrm{g} / \mathrm{ml})$ and red fluorescent protein overexpression. Puromycin resistant and red fluorescent protein-positive colonies were subsequently isolated, expanded, and screened for knockdown of TRPV3 by qPCR and functional assays. A single colony showing changes in TRPV3 expression and function was selected for the studies shown herein.

Statistical Analysis and Experimental Design. All experiments were designed to test a preplanned hypothesis, with the exception of studies evaluating different types of wood/biomass smoke PM (Supplemental Figs. 2 and 5), which were exploratory in nature. Data are represented as means \pm S.D. Significance was determined using ordinary one- or two-way ANOVA with Bonferroni correction at the $95 \%$ confidence level, unless otherwise stated.

\section{Results}

Pine WSPM Promotes Cytotoxicity and Activates TRPV3 and TRPA1 in Lobar HBECs. Lobar HBECs were treated with increasing concentrations of pine WSPM for 24 hours. Pine WSPM caused dose-dependent cytotoxicity with an $\mathrm{LD}_{50}$ of $\sim 18.8 \mu \mathrm{g} / \mathrm{cm}^{2}$ (Fig. 1A). Lobar cells were also treated with pine WSPM $\left(78 \mu \mathrm{g} / \mathrm{cm}^{2}\right)$ to assess TRP channel activation using a short-term (100-second) calcium flux assay (Fig. 1B). Small ( 10\% ionomycin) but significant changes in cytosolic calcium were observed immediately after WSPM treatment. This response was partially inhibited $(\sim 50 \%)$ by cotreating cells with the TRPA1 antagonist A967079 $(20 \mu \mathrm{M})$ and $~ 70 \%$ using $10 \mu \mathrm{M}$ TRPV3 antagonist. Figure 1B shows that both TRPA1 and TRPV3 are activated by pine WSPM in lobar HBECs, whereas Supplemental Figure 1 shows that neither A967079 nor the TRPV3 antagonist alone elicited calcium flux in these cells.

TRPA1 Primarily Localizes to the Cell Surface, Whereas TRPV3 is Expressed on the ER of HBECs. The subcellular localization of TRPA1 and TRPV3 in HBECs was not known, and locations could be critical for understanding mechanisms of cytotoxicity in HBECs treated with WSPM and agonists of these ion channels. Despite numerous attempts, TRPA1 localization by immunocytochemical methods was unsuccessful due to poor quality antibodies. Thus, TRPA1 localization was further evaluated using calcium flux assays and inhibitors of calcium influx versus intracellular/ER release (Fig. 2A). Calcium flux elicited by the selective TRPA1 agonist AITC was attenuated $>95 \%$ by cotreating cells with the extracellular calcium chelator EGTA and the cellimpermeable, nonselective TRP channel blocker, ruthenium red, as previously described (Thomas et al., 2007; DeeringRice et al., 2015). Control data for EGTA/ruthenium red are also shown in Supplemental Figure 1. Thapsigargin pretreatment (5 minutes), used to deplete $\mathrm{ER} \mathrm{Ca}^{2+}$, only partially $(\sim 50 \%)$ attenuated calcium flux. These results suggest that TRPA1 resides on both the cell surface and the ER of cells but that there may be a functional link between cell surface TRPA1 activation and ER calcium release.

Unlike AITC, calcium flux elicited by pine WSPM and carvacrol, which activate both TRPA1 and TRPV3, were only 

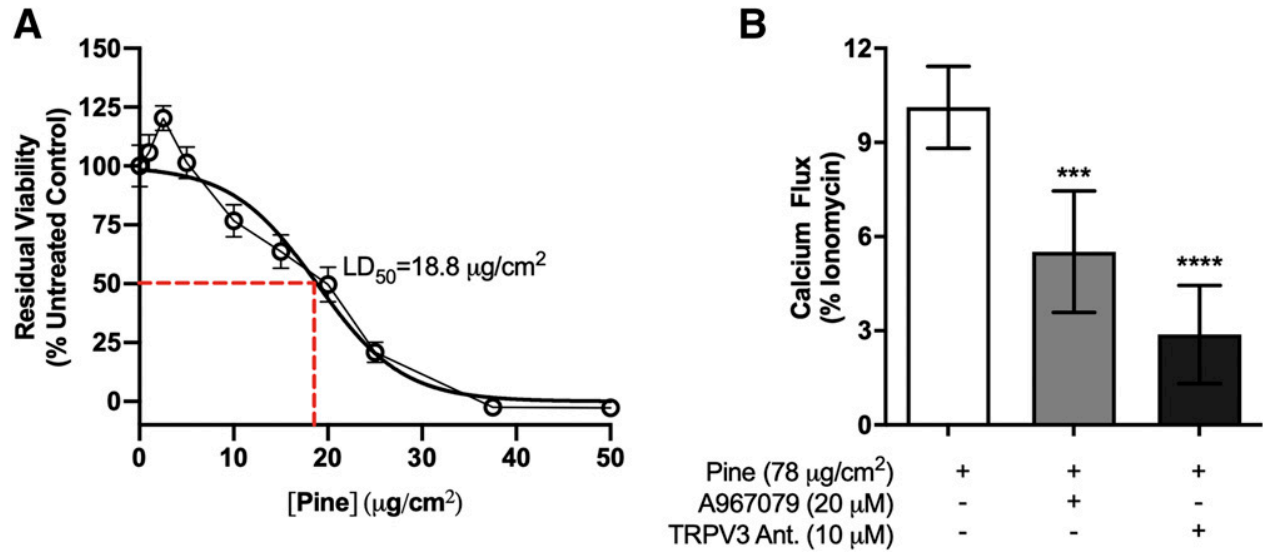

Fig. 1. WSPM cytotoxicity and the contributions of TRPV3 and TRPA1 in regulating pine WSPM-induced calcium flux. (A) Changes in lobar HBEC viability after 24-hour treatment with increasing concentrations of pine WSPM. Data are represented as mean percentages $( \pm$ S.D. $)$ of residual viability compared with untreated control for $n=3$ replicates. (B) Inhibition of pine WSPM-induced calcium flux in lobar HBECs treated with pine WSPM $\left(78 \mu \mathrm{g} / \mathrm{cm}^{2}\right) \mathrm{with}$ or without the TRPA1 antagonist A967079 $(20 \mu \mathrm{M})$ or the TRPV3 antagonist (TRPV3 Ant.; $10 \mu \mathrm{M})$. Calcium flux data were recorded over a 100-second period, and values for vehicle treatment were subtracted and then normalized to the maximum fluorescence change elicited by ionomycin $(10 \mu \mathrm{M})$. Data represent means \pm S.D. for $n \geq 3$ replicates. ${ }^{* * *} P<0.001 ;{ }^{* * * *} P<0.0001$ using an ordinary ANOVA with a Dunnett multiple comparisons test vs. pine WSPM only treatment.

partially attenuated by EGTA and ruthenium red cotreatment $(\sim 25 \%-50 \%)$ and to a much greater extent by thapsigargin pretreatment $(\sim 70 \%-90 \%)$ (Fig. 2A). These results suggest that TRPV3 may reside within cells and contribute to the release of ER calcium by pine WSPM and carvacrol.

Immunocytochemical analysis of TRPV3 in lobar HBECs revealed overlapping staining with the ER-specific biomarker calnexin (Fig. 2B; Supplemental Fig. 3A). In agreement with this pattern of staining, calcium flux elicited by the selective TRPV3 agonist drofenine $(250 \mu \mathrm{M}$; Fig. 2A) (Deering-Rice et al., 2014) was attenuated $\sim 85 \%$ with thapsigargin pretreatment but $<2 \%$ by EGTA and ruthenium red. These data suggest that the majority of TRPV3 is expressed on the ER, and the cumulative results are consistent with both TRPA1 and TRPV3 regulating calcium flux elicited by pine WSPM (as in Fig. 1B), whereby TRPA1 contributes mainly to the EGTA/ ruthenium red-sensitive (i.e., cellular influx) component, and both TRPA1 and TRPV3 regulate ER calcium release.

Pine WSPM Induces a Robust ERS Response in HBECs. Based on the finding that pine WSPM caused ER calcium release, HBECs were treated with pine WSPM $\left(20 \mu \mathrm{g} / \mathrm{cm}^{2}\right)$, and total mRNA was isolated at various times after treatment, to assess the expression of prototypical ERS-associated biomarkers; readers are directed to Scheme 1 as a reference for how each biomarker is involved in the overall integrated ERS response. Treated cells demonstrated marked time-dependent upregulation of the protein kinase R-like endoplasmic reticulum kinase (PERK/eIF2 $\alpha \mathrm{K} 3$ ) pathway biomarkers ATF3 and DDIT3, marked and transient upregulation of the chaperone HSPA1A, and a gradual and sustained increase in XBP1 mRNA splicing indicative of IRE1 $\alpha / \beta$ activation (Fig. 3). As expected, XBP1 splicing was rapid, occurring within 2 hours, followed by HSPA1A induction at 4 hours, whereas PERK-dependent induction of ATF3 and DDIT3 mRNA was delayed, occurring at times $>4$ hours. Induction of ERS, assessed using DDIT3 and ATF3 induction, by a variety of WSPM materials (Supplemental Fig. 2), as well as DDIT3 induction in HBEC3-KT cells (38 \pm 4 -fold) and SAECs $(6.23 \pm 0.06$-fold), was also observed after pine WSPM treatment, indicating that ERS is a common consequence of
A

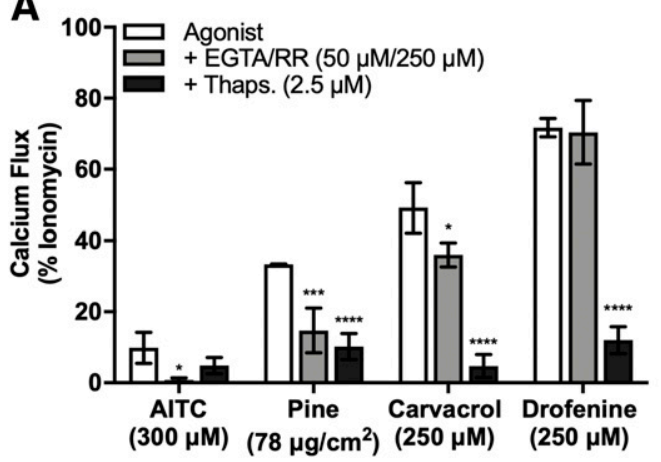

B

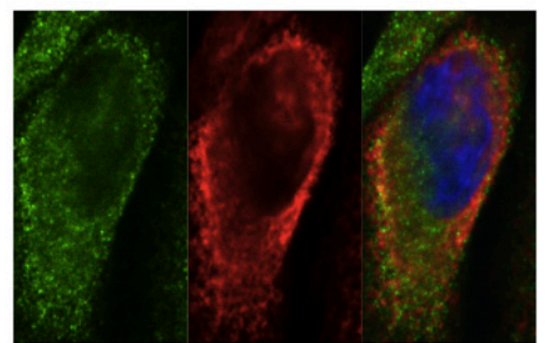

Fig. 2. Localization of TRPA1 and TRPV3 in HBECs. (A) Functional expression of TRPA1 and TRPV3 in lobar HBECs using a calcium flux assay. Agonists of TRPA1 (AITC, $300 \mu \mathrm{M}$ ), TRPA1/TRPV3 (pine WSPM, $78 \mu \mathrm{g} / \mathrm{cm}^{2}$, and carvacrol, $250 \mu \mathrm{M}$ ), and TRPV3 (drofenine, $250 \mu \mathrm{M}$ ) were used in combination with either the EGTA $(50 \mu \mathrm{M})$ and ruthenium red $(250 \mu \mathrm{M})$ to block the influx of extracellular calcium into cells, or thapsigargin (Thaps.; 2.5 $\mu \mathrm{M})$ to deplete intracellular/ER calcium stores. Calcium flux data (over a 100-second period) are represented as a percentage of the maximum fluorescence in the cells, elicited by the calcium ionophore ionomycin $(10 \mu \mathrm{M})$. Data are represented as means $\pm \mathrm{S} . \mathrm{D}$. from $n=3$ replicates. $* P<0.05$; *** $P<0.001 ; * * * * P<0.0001$ using ordinary two-way ANOVA and the Tukey post-test comparing each treatment to the respective agonist only control. (B) Representative immunostaining of TRPV3 (green), the ER biomarker calnexin (red), and nuclei (blue) in lobar HBECs. The complete fluorescence micrographs may be found in Supplemental Figure 3. 
A

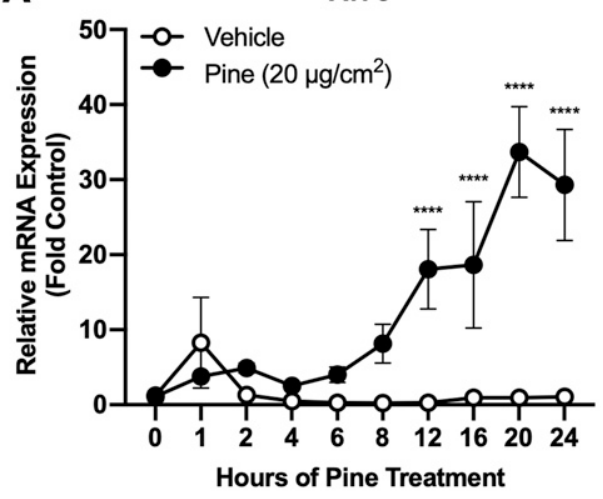

C

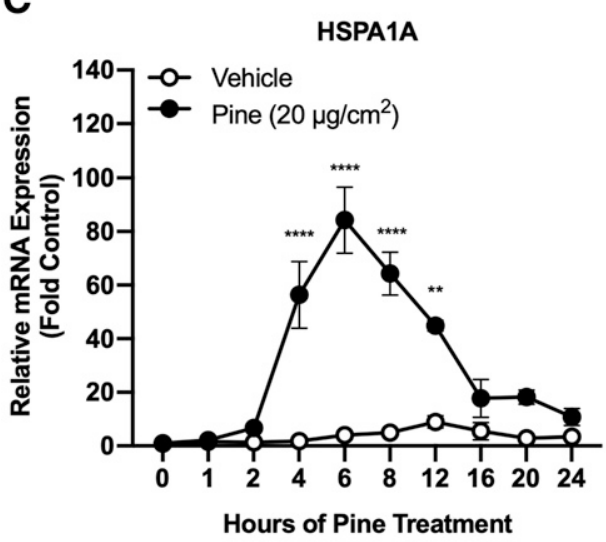

E

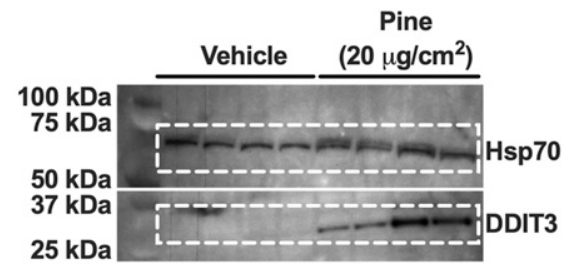

B

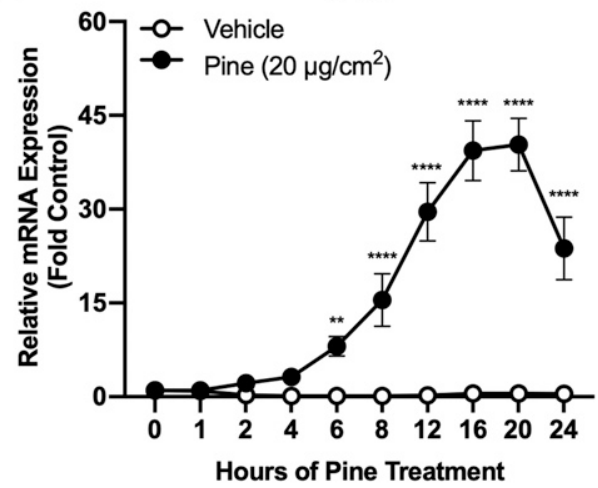

D

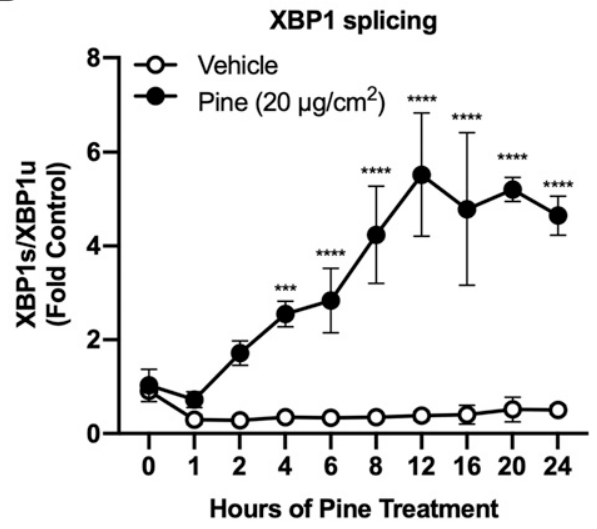

Fig. 3. Temporal profiling of ERS biomarkers in lobar HBECs in response to pine WSPM treatment. Expression of mRNA for the ERS biomarkers ATF3 (A), DDIT3 (B), HSPA1A (C), and spliced XBP1 (D) by lobar HBECs during treatment with pine WSPM $\left(20 \mu \mathrm{g} / \mathrm{cm}^{2}\right.$, filled circles) or vehicle (open circles) over time. Differences in the magnitude of induction should be noted. Each time point was normalized against the 0 -hour vehicletreated control and is presented as mean \pm S.D. from $n=3$ replicates. (E) Western blots confirming the upregulation of DDIT3 and HSP70 proteins in pine WSPM-treated HBECs. ATF 3 and TRPV3 were not detected by Western blot; recently purchased TRPV3 antibody aliquots retain selectivity but show marked decreases in sensitivity for detecting TRPV3 in lung cells. ${ }^{* *} P<0.01 ; * * * P<0.001$; $* * * * P<0.0001$. Statistical significance was determined using an ordinary twoway ANOVA using the Sidak multiple comparisons test. exposure of HBECs to WSPM, with the most potent TRPA1/ TRPV3 agonists generally producing the greatest effects.

TRPV3 and TRPA1 Activity Differentially Modulate Pine WSPM-Induced ERS. To determine the contributions of TRPA1 and TRPV3 to pine WSPM-induced ERS, cells were treated with pine WSPM $\left(20 \mu \mathrm{g} / \mathrm{cm}^{2}\right)$ in the absence or presence of TRPA1 or TRPV3 antagonists for 24 hours. Inhibition of TRPA1 with A967079 (20 $\mu \mathrm{M})$ partially $(\sim 30 \%-40 \%)$ attenuated ERS, measured using DDIT3, ATF3, and HSPA1A induction (Fig. 4, A-C). However, XBP1 splicing was slightly exacerbated (Fig. 4D). Similar results were observed using carvacrol, a TRPA1/TRPV3 agonist that mimics the activity of pine WSPM (Fig. 4). Alternatively, inhibition of TRPV3 generally resulted in an exacerbation of ERS using the same indices (Fig. 4). As shown in Supplemental Figure 4, similar trends for TRPA1 and TRPV3 inhibition were observed using coniferaldehyde (a component of pine WSPM) as a TRPA1 agonist, whereas neither antagonist universally affected cellular responses to drofenine, a selective TRPV3 agonist. Collectively, these results show that TRPA1 initiates ERS in response to pine WSPM and "chemical mimics" (i.e., carvacrol and coniferaldehyde) treatments, whereas TRPV3 attenuates these effects; TRPV3 activation alone had only minimal effects on ERS biomarker induction. These data suggest disparate roles for TRPA1 and TRPV3 in regulating ERS.

Pine WSPM Modulates TRPA1 and TRPV3 Expression during ERS. Lobar HBECs were treated with pine WSPM $\left(20 \mu \mathrm{g} / \mathrm{cm}^{2}\right)$ for various times up to 24 hours. Previous work has shown that lobar HBECs basally express TRPA1 at $\sim 10$ copies per 100,000 copies of $\beta 2 \mathrm{M}$ and TRPV3 at $\sim 110$ copies per 100,000 copies of $\beta 2 \mathrm{M}$ (Deering-Rice et al., 2018). Here, relative to the control cells in which a slight induction of TRPA1 was observed, TRPA1 transcripts remained low over time after pine WSPM treatment (Fig. 5A). Alternatively, TRPV3 transcripts were rapidly and transiently upregulated after pine WSPM treatment (up to $\sim 150$-fold greater than control) and remained elevated ( $\sim 20$-fold control cells) for up to 24 hours (Fig. 5B). We hypothesize that these changes in TRPA1 and TRPV3 may reflect adaptive changes in cells, representing a purposeful effort to decrease the deleterious effects of TRPA1 activation, while promoting TRPV3 function to counteract the procytotoxic effects associated with TRPA1 activation and pathologic ERS. Similar to ERS biomarkers, 
A
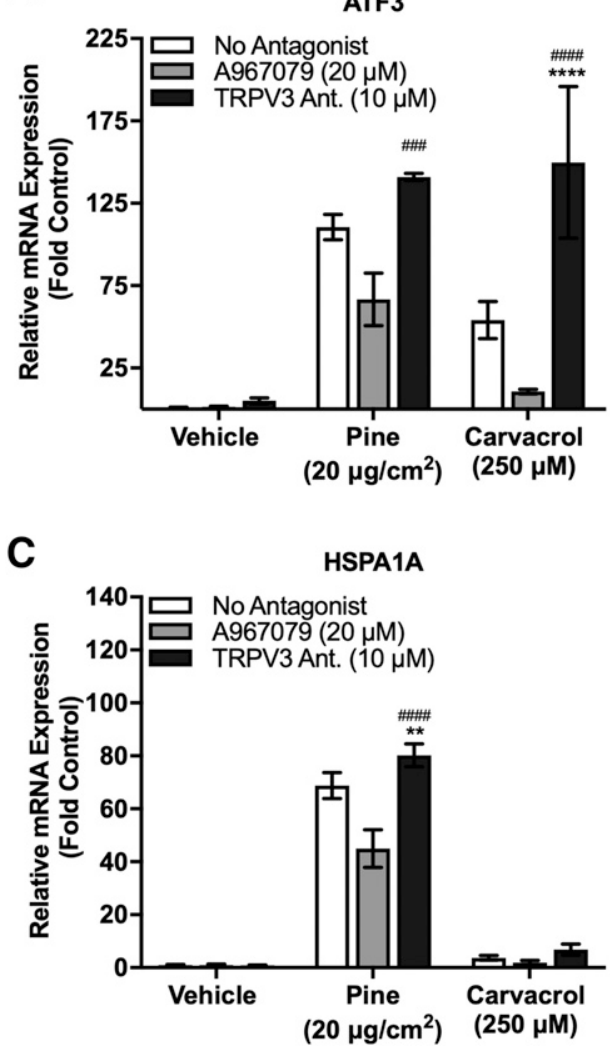

B

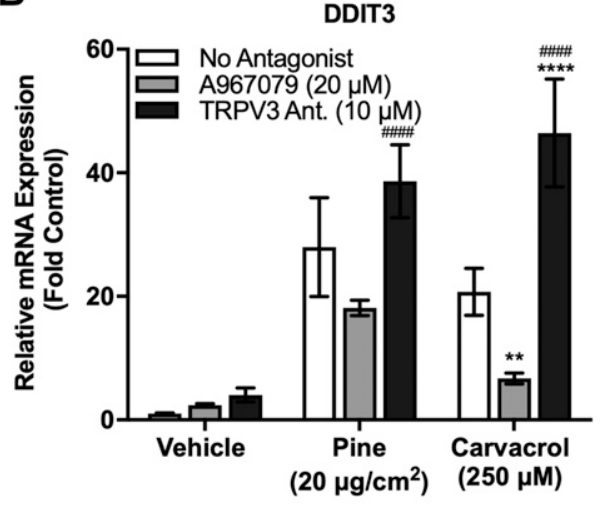

D

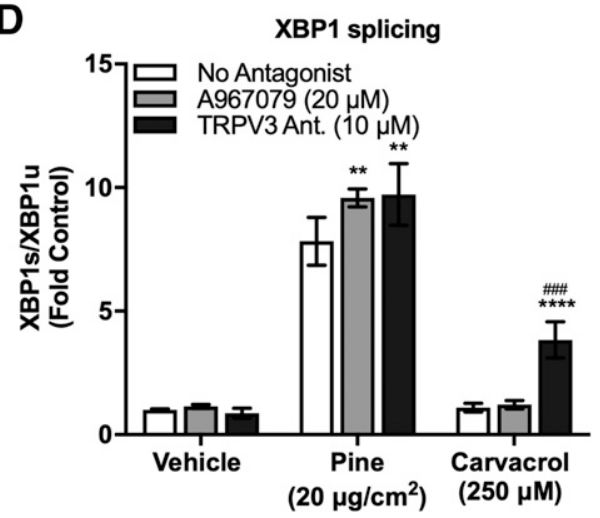

Fig. 4. Effects of TRPA1 or TRPV3 inhibition on pine WSPM-induced ERS biomarker induction. Expression of ATF3 (A), DDIT3 (B), HSPA1A (C), and spliced XBP1 (D) mRNA 24 hours after treatment of lobar HBECs with pine WSPM $\left(20 \mu \mathrm{g} / \mathrm{cm}^{2}\right)$ or carvacrol $(250 \mu \mathrm{M})$, in the presence of either the TRPA1 antagonist A967079 $(20 \mu \mathrm{M})$ or the TRPV3 antagonist (TRPV3 Ant.; $10 \mu \mathrm{M}$ ). Data were normalized to the vehicle-treated group and are represented as means \pm S.D. from $n=3$ replicates. Statistical significance was determined using an ordinary two-way ANOVA using the Tukey posttest comparing all treatments for each group. ${ }^{* *} P<0.01 ; * * * * P<0.0001$ relative to the respective no antagonist control. ${ }^{\# \# \#} P<0.001 ;{ }^{\# \# \# \#} P<0.0001$ indicate differences between the TRPA1 and TRPV3 antagonist groups.
TRPV3 transcripts were also upregulated by a variety of WSPM subtypes (Supplemental Fig. 5), as well as $19 \pm 2$-fold in HBEC3-KT cells treated with pine WSPM, suggesting again that this effect is a common response of HBECs to WSPM of various origins.

Overexpression of TRPV3 Confers Resistance to ERS in HBECs. To further understand the significance of TRPV3 induction during ERS, TRPV3OE cells were studied. BEAS$2 \mathrm{~B}$ and TRPV3OE cells were treated with increasing concentrations of pine WSPM $\left(1,5\right.$, and $\left.20 \mu \mathrm{g} / \mathrm{cm}^{2}\right)$ and a variety of prototypical ERS-inducing agents for 4 hours. The cells were then assayed for changes in mRNA for ERS biomarkers using qPCR (Table 1). Specifically, in response to the majority of the ERS-inducing agents tested, TRPV3OE cells exhibited reduced levels of DDIT3 and ATF3 induction, as well as decreased XBP1 splicing (XBP1s/XBP1u). Interestingly, HSPA1A induction appeared to be specifically associated with pine WSPM treatment. Collectively, these data imply a unique and broadly relevant role of TRPV3 in fettering the pathologic PERK-dependent ERS branch and presumably the associated pathologic endpoints.

TRPV3 Overexpression Alters ERS-Induced Cell Cycle Arrest. A hallmark of PERK activation during ERS is cell cycle arrest, particularly in the G2 phase. BEAS-2B and TRPV3OE cells were treated with thapsigargin $(1 \mu \mathrm{M})$ or pine WSPM (5 $\mu \mathrm{g} / \mathrm{cm}^{2} ; 0.011 \mathrm{mg} / \mathrm{ml}$ pine WSPM solution) for 4 hours and assayed for cell-cycle distribution by flow cytometry (Fig. 6). Both thapsigargin and pine WSPM caused cell cycle arrest at G2 in BEAS-2B cells. However, TRPV3OE cells exhibited a shift to $\mathrm{S}$-phase in response to both
A

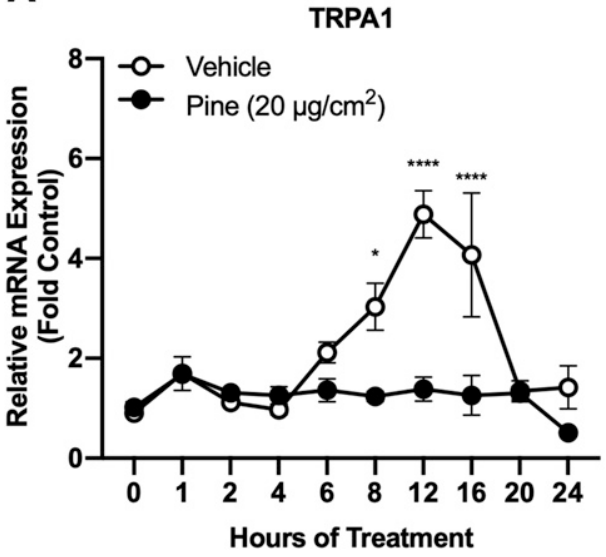

B

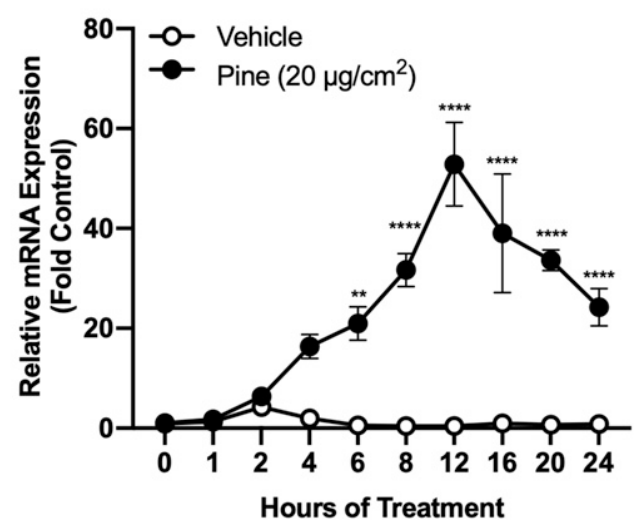

Fig. 5. Temporal changes in TRPA1 and TRPV3 mRNA expression after pine WSPM treatment. TRPA1 (A) and TRPV3 (B) mRNA expression over time in lobar HBECs during treatment with pine WSPM $\left(20 \mu \mathrm{g} / \mathrm{cm}^{2}\right.$; filled circles $)$ or vehicle (open circles). Each time point was normalized against the 0 hour vehicletreated control and is represented as mean \pm S.D. from $n=3$ replicates. *, $\mathrm{p}<0.05$; **, $\mathrm{p}<0.01$; ****, $\mathrm{p}<0.0001$. Statistical significance was determined using an ordinary two-way ANOVA using the Sidak multiple comparisons test. 


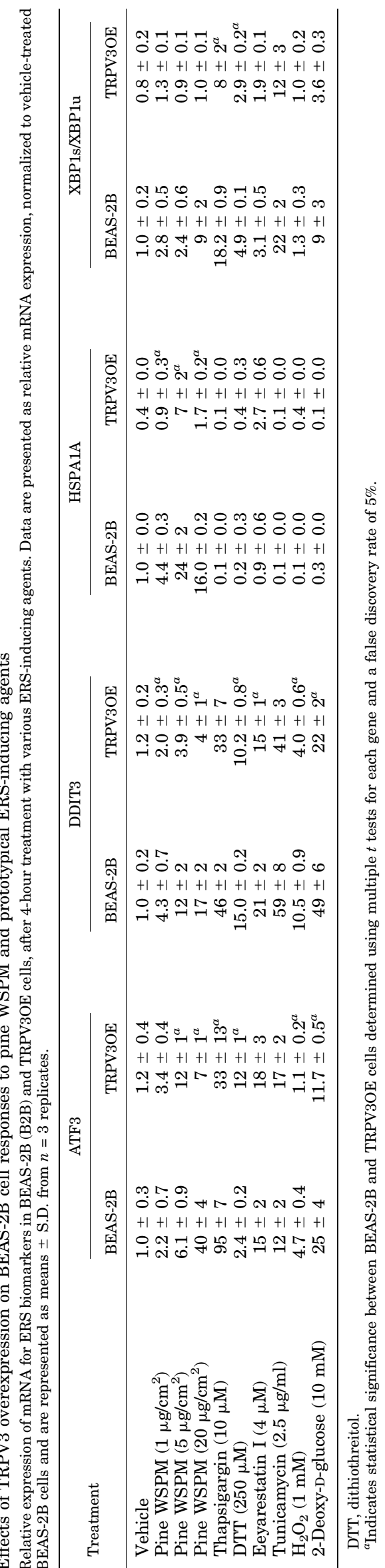

treatments. This shift away from arrest at G2 indicates a reduction in cell cycle arrest and a fundamental alteration in cellular programming associated with the PERK-driven pathologic, proapoptotic branch of the ERS response.

TRPV3 Knockdown Sensitizes Cells to ERS Elicited by Pine WSPM. To further explore the hypothesis that TRPV3 is protective in the context of pathologic ERS and pneumotoxic WSPM expsoure, BEAS-2B cells stably overexpressing shRNA targeting TRPV3 or a "scrambled" control shRNA were treated with either vehicle or pine WSPM at $20 \mu \mathrm{g} / \mathrm{cm}^{2}$ for 4 hours, or $10 \mu \mathrm{g} / \mathrm{cm}^{2}$ for 20 hours; doses were adjusted slightly to account for differences in sensitivity of BEAS-2B cells versus lobar HBECs to the pine WSPM. The scrambled shRNA control BEAS-2B cells basally expressed $4.1 \pm 0.7$ copies of TRPV3 per 100,000 copies of $\beta 2 \mathrm{M}$. As expected, TRPV3 mRNA was reduced $\sim 50 \%-60 \%$ in the TRPV3 shRNA knockdown cells, to levels essentially at the limit of detection of the qPCR assay (Fig. 7, A and $\mathrm{C}$ ). Furthermore, in control cells, pine WSPM induced TRPV3 mRNA expression, which was not observed in the TRPV3 shRNAknockdown cells at 4 hours and was attenuated $\sim 50 \%$ at 20 hours. Finally, similar to the pharmacological inhibition data, shRNA-mediated knockdown of TRPV3 exacerbated ERS, measured using DDIT3 induction as an indicator of pathologic ERS at 4 and 20 hours after pine WSPM treatment (Fig. 7, B and D).

Effects of TRPA1 and TRPV3 Inhibition on WSPM Cytotoxicity. An ultimate goal of this study was to determine how and to what extent TRPA1 and TRPV3 regulated the cytotoxic effects of pneumotoxic WSPM in primary HBECs. As such, the effects of TRPA1 and TRPV3 inhibition were evaluated as inhibitors of cytotoxicity. Lobar HBECs were treated with pine WSPM at $20 \mu \mathrm{g} / \mathrm{cm}^{2}$ for 24 hours with either the TRPA1 antagonist A967079 $(20 \mu \mathrm{M})$ or the TRPV3 antagonist $(10 \mu \mathrm{M})$; higher concentrations of the antagonists were tested, but as per prior publications by our group, the antagonists themselves became cytotoxic (Deering-Rice et al., 2014, 2018; Memon et al., 2020). Consistent with the cumulative findings on the modulation of pathologic ERS by TRPA1 and TRPV3 activity, the cytotoxicity of pine WSPM was blocked by cotreating cells with the TRPA1 antagonist A967079 $(20 \mu \mathrm{M})$ but was exacerbated ( 2-fold) by TRPV3 inhibition (Fig. 8). These findings demonstrate that TRPA1 and TRPV3 differentially regulate pathologic ERS in HBECs, ultimately influencing the ability of cells to either adapt to and survive stress, or succumb to stress and die.

Effects of TRPA1 and TRPV3 Inhibitors on Calcium Flux. Finally, to clarify the differential effects of TRPA1 and TRPV3 on ERS and cytotoxicity, the effects of the TRPA1 and TRPV3 antagonists on TRPA1- and TRPV3-driven calcium flux in both lobar HBECs and TRPA1 and TRPV3overexpressing HEK-293 cells were tested after treatment with selective agonists of each channel. In lobar HBECs, TRPA1 inhibition by A967079 $(20 \mu \mathrm{M})$ attenuated both TRPA1-driven calcium flux $(\sim 25 \%-40 \%)$ by AITC $(150 \mu \mathrm{M}$; Fig. 9A) and TRPV3-driven calcium flux ( 85\%-90\%) by drofenine (250 $\mu \mathrm{M}$; Fig. 9B). However, TRPV3 inhibition by the TRPV3 antagonist $(10 \mu \mathrm{M})$ attenuated only TRPV3-driven calcium flux $(\sim 50 \%-60 \%)$ by drofenine $(250 \mu \mathrm{M}$; Fig. 9B) while enhancing ( $\sim$-fold) TRPA1-driven calcium flux by AITC (150 $\mu \mathrm{M}$; Fig. 9, A and B). The effects of these antagonists were unique to HBEC cells, based on the finding that neither 
A

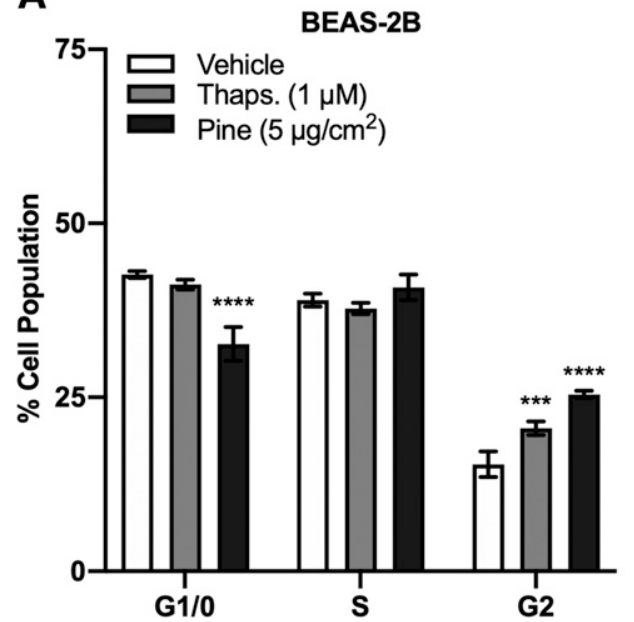

B

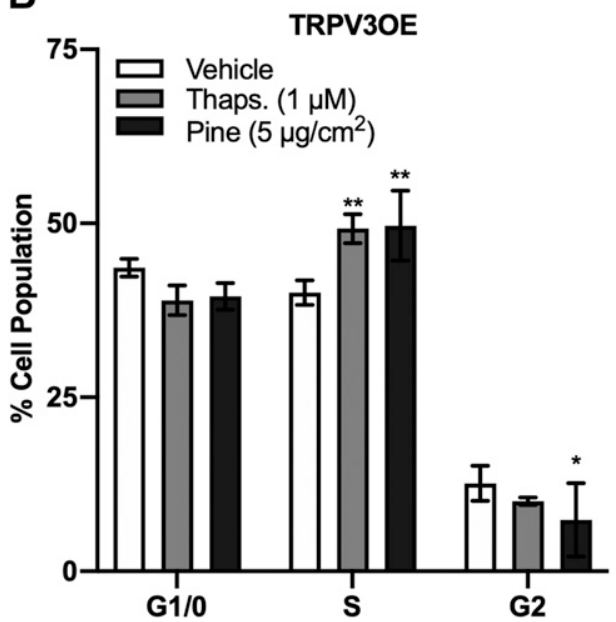

Fig. 6. Effect of TRPV3 overexpression on ERS-associated cell cycle arrest. Cell cycle analysis by flow cytometry on BEAS2B cells (A) or TRPV3OE cells (B), after 4 hours treatment with either vehicle (white bars), thapsigargin (Thaps.; $1 \mu \mathrm{M}$; black bars), or pine WSPM ( $5 \mu \mathrm{g} / \mathrm{cm}^{2}$; gray bars). The data represent cells in a specific phase of the cell cycle as a percentage of total viable cells. Data are presented as means \pm S.D. from $n=3$ replicates. $* P<$ $0.05 ; * * P<0.01 ; * * * P<0.001 ; * * * * P<$ 0.0001 . Statistical significance was determined using an ordinary two-way ANOVA with the Bonferonni correction. antagonist affected the other channel in TRPA1 or TRPV3overexpressing HEK-293 cells (Fig. 9, C and D). Additionally, TRPA1 activation by a nonelectrophilic agonist, 2,4 ditertbutylphenol, was blocked by A967079 $(20 \mu \mathrm{M})$ in TRPA1overexpressing cells, indicating that the effects of A967079 on TRPA1 activity are independent of the agonist reactivity or mechanism of binding (Supplemental Fig. 6). These results imply a functional coupling between TRPA1 and TRPV3 activities in HBECs, wherein TRPA1 stimulates TRPV3 and TRPV3 attenuates TRPA1, seemingly explaining the differential effects that TRPA1 and TRPV3 have on ERS and cytotoxicity.

\section{Discussion}

The goals of this study were to further define mechanisms by which pneumotoxic WSPM affect HBEC viability using a primary lung cell model and to elucidate roles for TRPA1 and TRPV3 in regulating ERS and cell death caused by WSPM,
A

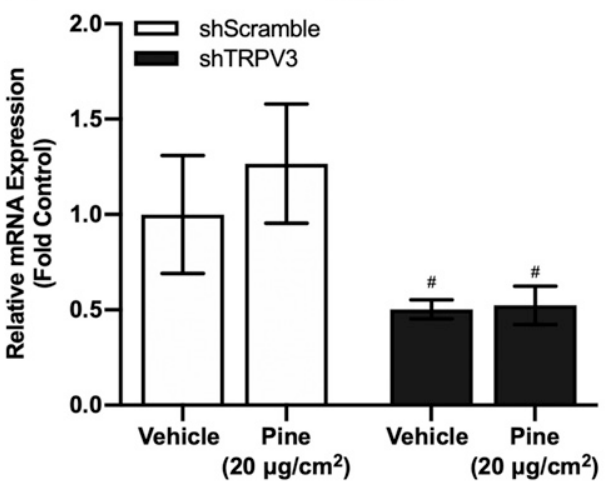

4 hour treatment

C

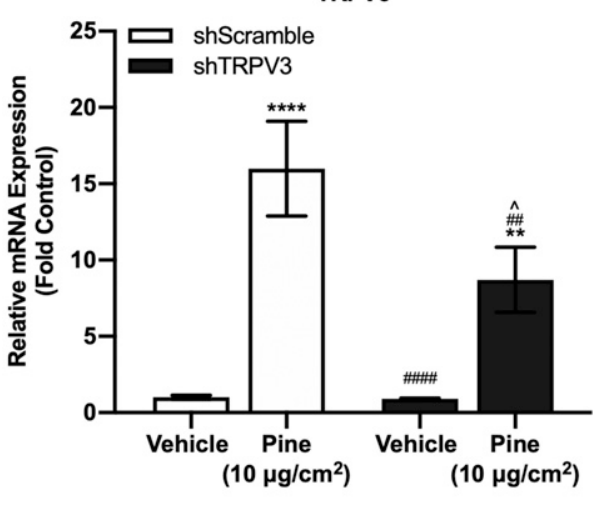

20 hour treatment

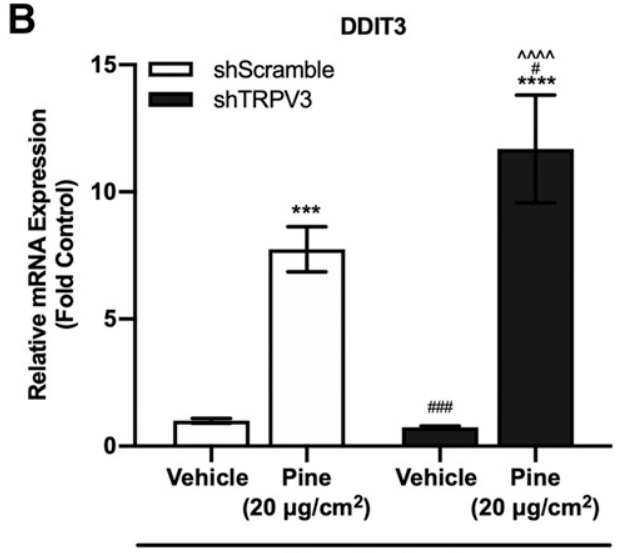

4 hour treatment

D

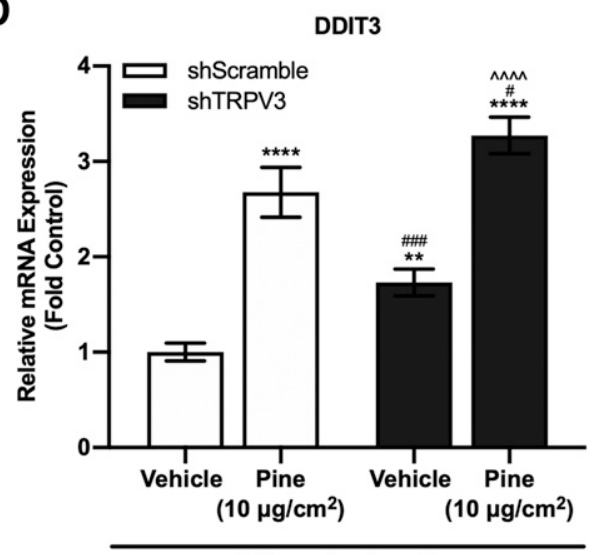

20 hour treatment
Fig. 7. Effects of shRNA-driven TRPV3 knockdown on the sensitivity of BEAS-2B cells to pine WSPM-induced ERS. mRNA expression of TRPV3 (A and $\mathrm{C}$ ) and DDIT3 (B and D) in BEAS-2B cells stably overexpressing control/scrambled shRNA (shScramble; white bars) or shRNA targeting TRPV3 (shTRPV3; black bars) after 4-hour treatment with vehicle and pine WSPM $\left(20 \mu \mathrm{g} / \mathrm{cm}^{2}\right)(\mathrm{A}$ and B) or 20 hour treatment with vehicle and pine WSPM $\left(10 \mu \mathrm{g} / \mathrm{cm}^{2}\right)(C$ and D). Data were normalized to the vehicle-treated shTRPV3 cells and are presented as means \pm S.D. from $n=3$ replicates. $* * P<0.01 ; * * * P<0.001 ; * * * * P<$ 0.0001 compared with vehicle treated control cells, ${ }^{\#} P<0.05$; ${ }^{\# \#} P<0.01$; ${ }^{\# \# \# P} P<0.001 ;{ }^{\# \# \# \#<0.0001 \text { compared }}$ with pine WSPM treated control cells, and $\wedge P<0.05 ; \wedge \wedge \wedge \wedge P<0.0001$ compared with vehicle-treated scramble control cells using an ordinary two-way ANOVA and the Tukey post-test comparing all data sets. 


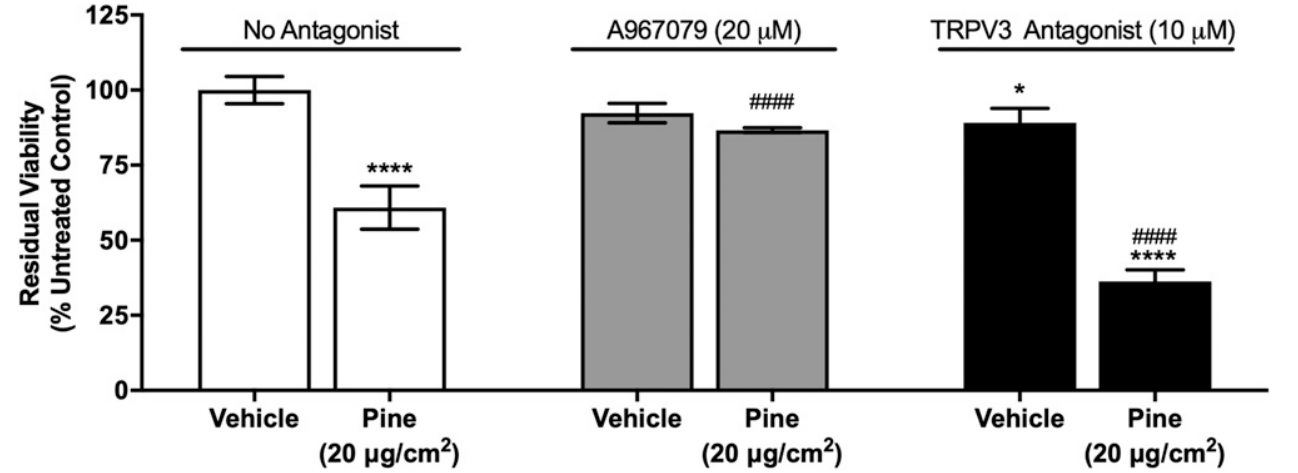

Fig. 8. Effects of TRPA1 and TRPV3 inhibition on pine WSPM-induced cytotoxicity. Lobar HBEC viability was assayed after 24 hours of treatment with pine WSPM $\left(20 \mu \mathrm{g} / \mathrm{cm}^{2}\right)$, in the presence of A967079 (20 $\mu \mathrm{M}$, gray bars $)$ or the TRPV3 antagonist $(10 \mu \mathrm{M}$, black bars). Data were normalized against the vehicle control and are presented as means \pm S.D. for $n=3$ replicates. $* P<0.05 ; * * * * P<0.0001$ compared with vehicle treated cells with-

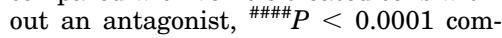
pared with pine WSPM treatment without antagonists using an ordinary two-way ANOVA and the Dunnett posttest. using pine WSPM as a model. This study identified differences in the subcellular localization and functional significance of TRPA1 and TRPV3, both in the context of initiation of pathologic ERS and the manifestation of cytotoxicity, revealing atypical and contrasting roles. Specifically, TRPA1 activation was found to initiate pathologic, PERK-driven ERS, indicated by increases in the expression of established biomarkers of this pathway (i.e., proapoptotic DDIT3 and ATF3), cell cycle arrest at G2, and cell death. In contrast, a novel and unexpected role for TRPV3 as a negative regulator of PERKdependent pathologic ERS after pine WSPM, TRPA1 agonist, and prototypical ERS-inducing agent treatments was revealed. Finally, changes in TRPA1 and TRPV3 mRNA expression were observed in HBECs after treatment with pine PM and multiple other forms of WSPM, highlighting a potential common mechanism by which pneumotoxic substances that either directly activate TRPA1 or TRPV3 and/or produce ERS might affect HBECs. Consistent with this idea, we have previously hypothesized that differences in the relative abundance of TRPA1 and TRPV3 agonists in certain samples of diesel exhaust particle emissions (i.e., 2,4 ditertbutylphenol) may influence the relative proinflammatory potential of these materials in HBECs and animal models (Deering-Rice et al., 2019).

The specific contributions of TRPA1 and TRPV3 in regulating ERS and cytotoxicity were initilally puzzling. It was found
A

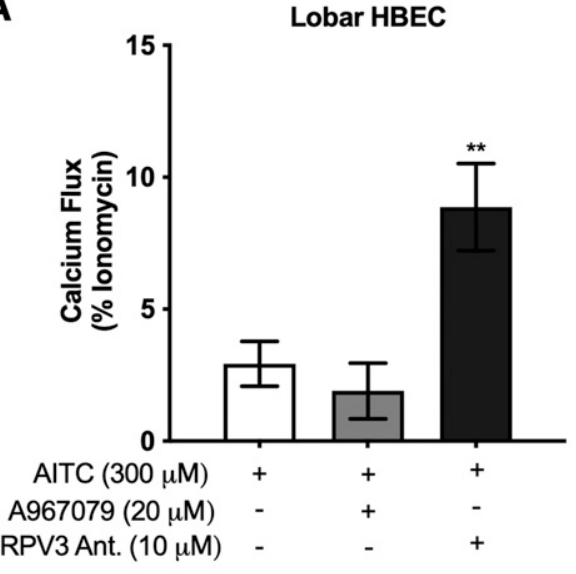

C

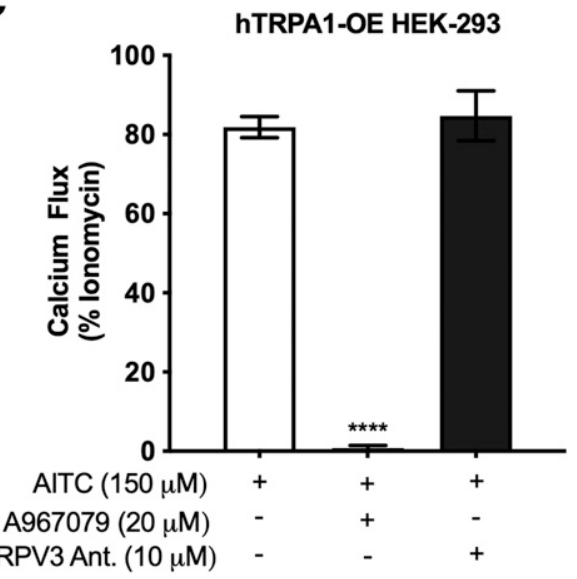

B

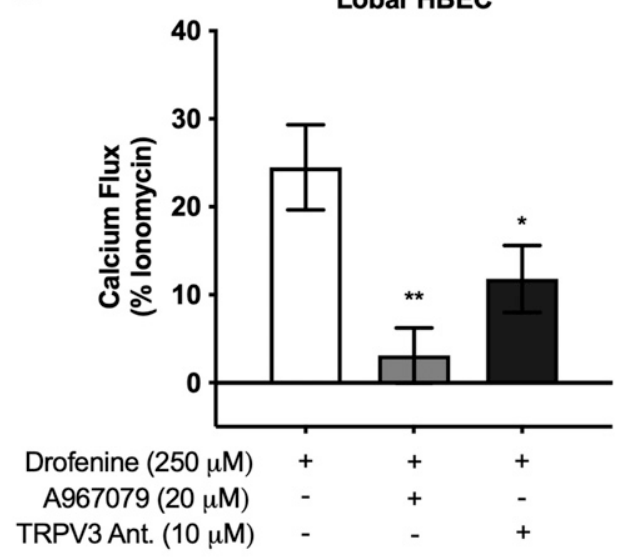

D

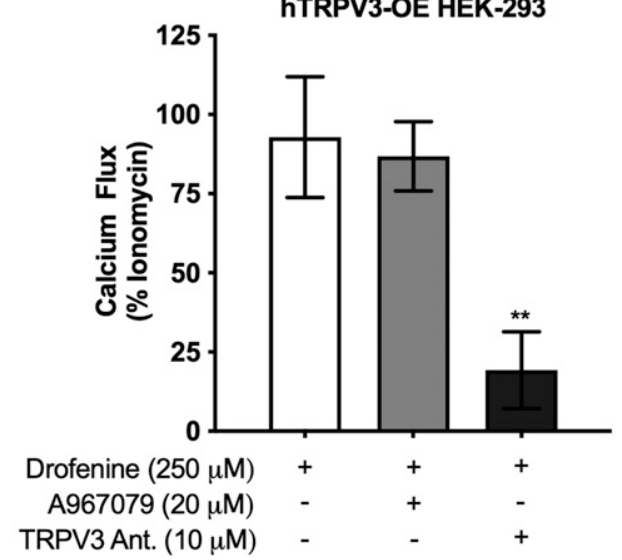

Fig. 9. Functional interactions between TRPA1 and TRPV3 activities. (A) Alterations in TRPA1-mediated calcium flux in lobar HBECs treated with AITC $(150 \mu \mathrm{M})$ with or without the TRPA1 antagonist A967079 $(20 \mu \mathrm{M})$ or the TRPV3 antagonist (TRPV3 Ant.; $10 \mu \mathrm{M}$ ). (B) Inhibition of TRPV3-mediated calcium flux in lobar HBECs treated with drofenine $(250 \mu \mathrm{M})$ with or without the TRPA1 antagonist A967079 $(20 \mu \mathrm{M})$ or a TRPV3 antagonist $(10 \mu \mathrm{M})$. Data are presented as means \pm S.E.M. from $n \geq 5$ replicates. (C) Effects of the TRPA 1 and TRPV3 antagonists on TRPA1-mediated calcium flux elicited byAITC $(150 \mu \mathrm{M})$ in TRPA1-overexpressing HEK-293 cells (hTRPA1-OE HEK-293). (D) Effects of the TRPA1 and TRPV3 antagonists on TRPV3-mediated calcium flux by drofenine $(250 \mu \mathrm{M})$ in TRPV3overexpressing HEK-293 cells. Data are presented as means \pm S.D. from $n \geq 3$ replicates. ${ }^{*} P<0.05 ; * * P<0.01 ;$ using an ordinary one-way ANOVA with the Bonferroni correction. 
that TRPA1 localized to both the cell surface and ER, whereas the majority of TRPV3 was associated with the ER. Yet, only TRPA1 was ultimately found to initiate pathologic ERS and cytotoxicity caused by pine WSPM and other TRPA1 agonists, whereas TRPV3 seemed to conteract this effect, despite being activated by pine WSPM, and the selective TRPV3 agonist drofenine, which generally did not induce a robust ERS response despite triggering $\mathrm{ER}$ calcium release. Influx of calcium into cells from extracellular sources is not typically associated with ERS. Thus, it is likely that the ER subpopulation of TRPA1 selectively drives the ERS responses observed after treatment with WSPM and other TRPA1 agonists. It is also possible that cell surface TRPA1 activation may promote ER calcium release through other ER calcium release channels such as ryanodine receptors and/or inositol triphosphate receptors, as suggested by others (Pan et al., 2016; Xu et al., 2017), to promote ERS. The finding that AITCdriven calcium flux in HBEC cells was only partially inhibited by thapsigargin pretreatment, but extensively inhibited by EGTA and ruthenium red, supports such a possibility.

Another hypothesis directly supported by the experimental data are that TRPA1 activation in HBECs stimulates TRPV3 activity to enhance ER calcium release, which in combination would explain the robust ERS responses observed with the WSPM and carvacrol, relative to more selective TRPA1 (i.e., AITC and coniferaldehyde) or TRPV3 (drofenine) agonists. Sensitization of TRPV3 by cytosolic calcium has been described, resulting from a reduction in calmodulin binding, which normally inhibits the channel (Nilius et al., 2014). Accordingly, pine WSPM-induced increases in cytosolic calcium via TRPA1 may sensitize TRPV3, which in turn would enhance ER calcium depletion and trigger a robust ERS response, as observed for carvacrol and pine WSPM, but not drofenine or coniferaldehyde. High intracellular calcium would also promote TRPA1 desensitization to temper TRPA1 activity (Ruparel et al., 2011; Memon et al., 2020), TRPV3 activity, and ultimately ERS, seemingly explaining how TRPV3 can reduce ERS caused by TRPA1 and TRPA1/TRPV3 agonists (Scheme 2).

Another intriguing finding was that the inhibitory effect of TRPV3 overexpression on ERS was not only limited to pine WSPM or TRP channel agonist treatment, suggesting that TRPV3 may play previously unrecognized roles in regulating the toxicity of a variety of agents. Specifically, TRPV3OE cells exhibited diminished pathologic ERS in comparison with BEAS-2B cells, after treatment with multiple distinct ERSinducing agents including an ER calcium depleting agent (thapsigargin), an inhibitor of glucose metabolism (2-deoxyD-glucose), oxidative and reductive stress-inducing agents $\left(\mathrm{H}_{2} \mathrm{O}_{2}\right.$ and dithiothreitol), a glycosylation inhibitor (tunicamycin), and the UPR/proteasomal inhibitor (Eeyarestatin-I). In general, TRPV3 overexpression conferred protection against ERS induced by most of these agents, based on decreases in the level of DDIT3 induction (i.e., the proapoptotic branch of the ERS response). These data imply a possible selective effect of TRPV3 on PERK-dependent signaling, which may result from TRPV3 promoting calcium homeostasis, as has been suggested for TRP canonical-1 in salivary gland cells (Sukumaran et al., 2019). Accordingly, the induction of TRPV3 in conjunction with suppressed TRPA1 expression may indicate a deliberate process by which cells attempt to restore the balance of ER and cytosolic calcium concentrations to limit ERS and other pathologic processes, wherein TRPA1 and TRPV3 play central roles. Regardless of the precise mechanism by which TRPV3 protects cells against ERS, it is clear that TRPV3 does protect cells against the deleterious effects of initiating a robust and prolonged ERS response that leads to cell cycle arrest and cytotoxicity.

Finally, regarding the primary conclusions of this study, our group previously reported that TRPV3 overexpression sensitized BEAS-2B cells to WSPM-induced cytotoxicity and that cells could be protected using a TRPV3 antagonist (DeeringRice et al., 2018). As such, the inhibitory roles of TRPV3 in pathologic ERS and exacerbation of cell death observed here contradict our initial hypothesis that TRPV3 would promote WSPM toxicity through an ERS-dependent mechanism. Primary HBECs are a more relevant model of the human airway epithelium, and the collective data reported here suggest that our initial hypothesis about TRPV3, based on TRPV3OE cells, was simply incorrect, and several key differences between TRPV3OE and normal cells may explain this discrepency. TRPV3-overexpressing cells lack the ability to control TRPV3 expression during stress. As such, the levels of TRPV3 in the TRPV3OE cells are supraphysiological and static. This is not without consequence, and we have observed a downregulation in the basal levels of key ERS response genes in the TRPV3OE cells in transcriptome analyses, suggesting that downregulating ERS machinery may limit the ability of cells to engage early cellular defenses against stress and to adapt to and compensate for and overcome the stress. Additionally, TRPA1 activation by AITC was not observed with TRPV3OE cells despite expressing higher levels of mRNA than normal BEAS$2 \mathrm{~B}$ and primary HBECs. As per Scheme 2, this may be due to calcium-dependent inhibition of TRPA1 by basal TRPV3 activity, rendering TRPA1 essentially incapable of initiating ERS. Finally, a portion of TRPV3 is expressed on the cell surface of TRPV3OE cells. Consequently, TRPV3OE cells likely die via a mechanism independent of ERS when treated with WSPM, which is not replicated in primary HBECs.

To summarize, this study provides new mechanistic insights into how pneumotoxic WSPM, agonists of TRPA1, and possibly other toxic agents can adversely affect HBECs. These formative findings are among the first to describe such roles for TRPA1 and TRPV3 in HBECs and provide new insights into both normal physiologic and pathophysiological functions associated with these receptors in the lung epithelium, particularly in the context of WSPM-induced lung cell injury. Significant results include the identification of seemingly opposing roles for TRPA1 and TRPV3 in regulating ERS and cell death in response to pine WSPM, and that TRPV3 may be critical in limiting ERS independent of the stimulus via coordinated induction during ERS. Finally, the protective effect of TRPV3 appears to be through activity-dependent inhibition of TRPA1 and specific modulation of the PERKdependent proapoptotic branch of the ERS signaling network involving DDIT3. Although additional work is needed to determine the broader significance of this signaling nexus in lung injury, these findings advance our general understanding of how these TRP channels regulate lung cell responses to toxicants such as WSPM.

\section{Acknowledgments}

The authors acknowledge the DNA/Peptide Core of the University of Utah Health Sciences Cores for synthesizing and high performance 
liquid chromatography-purifying primers and probes, as well as the Synthetic and Medicinal Chemistry Core for synthesizing the TRPV3 antagonist. The authors would also like to thank Katherine Rose for technical assistance with this work and Dr. Kevin D. Welch of the US Department of Agriculture Agricultural Research Services in Logan, UT, for assistance in obtaining the animal feces samples.

\section{Authorship Contributions}

Participated in research design: Nguyen, Memon, Burrell, DeeringRice, Reilly.

Conducted experiments: Nguyen, Memon, Burrell, AlmesticaRoberts, Rapp, Sun, Scott, Deering-Rice, Reilly.

Performed data analysis: Nguyen, Memon, Almestica-Roberts, Rapp, Sun, Deering-Rice, Rower, Reilly.

Wrote or contributed to the writing of the manuscript: Nguyen, Memon, Burrell, Almestica-Roberts, Rapp, Scott, Deering-Rice, Rower, Reilly.

\section{References}

Andrè $\mathrm{E}$, Campi B, Materazzi S, Trevisani M, Amadesi S, Massi D, Creminon C, Vaksman N, Nassini R, Civelli M, et al. (2008) Cigarette smoke-induced neurogenic inflammation is mediated by $\alpha, \beta$-unsaturated aldehydes and the TRPA1 receptor in rodents. J Clin Invest 118:2574-2582.

Bessac BF, Sivula M, von Hehn CA, Escalera J, Cohn L, and Jordt SE (2008) TRPA1 is a major oxidant sensor in murine airway sensory neurons. J Clin Invest 118:1899-1910.

Deering-Rice CE, Johansen ME, Roberts JK, Thomas KC, Romero EG, Lee J, Yost GS, Veranth JM, and Reilly CA (2012) Transient receptor potential vanilloid-1 (TRPV1) is a mediator of lung toxicity for coal fly ash particulate material. Mol Pharmacol 81:411-419.

Deering-Rice CE, Memon T, Lu Z, Romero EG, Cox J, Taylor-Clark T, Veranth JM, and Reilly CA (2019) Differential activation of TRPA1 by diesel exhaust particles: relationships between chemical composition, potency, and lung toxicity. Chem Res Toxicol 32:1040-1050.

Deering-Rice CE, Mitchell VK, Romero EG, Abdel Aziz MH, Ryskamp DA, Križaj D, Gopal VR, and Reilly CA (2014) Drofenine: A 2-APB analogue with greater selectivity for human TRPV3. Pharmacol Res Perspect 2:e00062.

Deering-Rice CE, Nguyen N, Lu Z, Cox JE, Shapiro D, Romero EG, Mitchell VK, Burrell KL, Veranth JM, and Reilly CA (2018) Activation of TRPV3 by wood smoke particles and roles in pneumotoxicity. Chem Res Toxicol 31:291-301.

Deering-Rice CE, Romero EG, Shapiro D, Hughen RW, Light AR, Yost GS, Veranth JM, and Reilly CA (2011) Electrophilic components of diesel exhaust particles (DEP) activate transient receptor potential ankyrin-1 (TRPA1): a probable mechanism of acute pulmonary toxicity for DEP. Chem Res Toxicol 24:950-959.

Deering-Rice CE, Shapiro D, Romero EG, Stockmann C, Bevans TS, Phan QM, Stone BL, Fassl B, Nkoy F, Uchida DA, et al. (2015) Activation of transient receptor potential ankyrin-1 by insoluble particulate material and association with asthma Am J Respir Cell Mol Biol 53:893-901.

Deering-Rice CE, Stockmann C, Romero EG, Lu Z, Shapiro D, Stone BL, Fassl B, Nkoy F, Uchida DA, Ward RM, et al. (2016) Characterization of Transient Receptor Potential Vanilloid-1 (TRPV1) variant activation by coal fly ash particles and associations with altered Transient Receptor Potential Ankyrin-1 (TRPA1) expression and asthma. J Biol Chem 291:24866-24879.

Ghio AJ, Soukup JM, Case M, Dailey LA, Richards J, Berntsen J, Devlin RB, Stone S, and Rappold A (2012) Exposure to wood smoke particles produces inflammation in healthy volunteers. Occup Environ Med 69:170-175.

Kim YH, Warren SH, Krantz QT, King C, Jaskot R, Preston WT, George BJ, Hays MD, Landis MS, Higuchi M, et al. (2018) Mutagenicity and lung toxicity of smoldering vs. Flaming emissions from various biomass fuels: implications for health effects from wildland fires. Environ Health Perspect 126:017011.

Korfei M, Ruppert C, Loeh B, Mahavadi P, and Guenther A (2016) The role of Endoplasmic Reticulum (ER) stress in pulmonary fibrosis. Endoplam Reticul Stress Dis 3:16-49.

Laumbach RJ and Kipen HM (2012) Respiratory health effects of air pollution: update on biomass smoke and traffic pollution. J Allergy Clin Immunol 129:3-11, NaN-13.

Liu JC, Wilson A, Mickley LJ, Dominici F, Ebisu K, Wang Y, Sulprizio MP, Peng RD, Yue X, Son JY, et al. (2017) Wildfire-specific fine particulate matter and risk of hospital admissions in urban and rural counties. Epidemiology 28:77-85.
Maiuolo J, Bulotta S, Verderio C, Benfante R, and Borgese N (2011) Selective activation of the transcription factor ATF6 mediates endoplasmic reticulum proliferation triggered by a membrane protein. Proc Natl Acad Sci USA 108: $7832-7837$.

Martino MB, Jones L, Brighton B, Ehre C, Abdulah L, Davis CW, Ron D, O’Neal WK, and Ribeiro CMP (2013) The ER stress transducer IRE1 $\beta$ is required for airway epithelial mucin production. Mucosal Immunol 6:639-654.

Memon TA, Nguyen ND, Burrell KL, Scott AF, Almestica-Roberts M, Rapp E, Deering-Rice CE, and Reilly CA (2020) Wood smoke particles stimulate MUC5AC overproduction by human bronchial epithelial cells through TRPA1 and EGFR signaling. Toxicol Sci 174:278-290 Available from: 10.1093/toxsci/kfaa006.

Nilius B, Bíró T, and Owsianik G (2014) TRPV3: time to decipher a poorly understood family member!. J Physiol 592:295-304.

Olloquequi J and Silva O R (2016) Biomass smoke as a risk factor for chronic obstructive pulmonary disease: effects on innate immunity. Innate Immun 22: 373-381.

Pan Y, Zhao G, Cai Z, Chen F, Xu D, Huang S, Lan H, and Tong Y (2016) Synergistic effect of ferulic acid and Z-ligustilide, major components of A. sinensis, on regulating cold-sensing protein TRPM8 and TPRA1 in vitro. Evid Based Complement Alternat Med 2016:3160247.

Reid CE, Jerrett M, Tager IB, Petersen ML, Mann JK, and Balmes JR (2016) Differential respiratory health effects from the 2008 northern California wildfires: a spatiotemporal approach. Environ Res 150:227-235

Ruparel NB, Patwardhan AM, Akopian AN, and Hargreaves KM (2011) Desensitization of transient receptor potential ankyrin 1 (TRPA1) by the TRP vanilloid 1-selective cannabinoid arachidonoyl-2 chloroethanolamine. Mol Pharmacol 80:117-123.

Shapiro D, Deering-Rice CE, Romero EG, Hughen RW, Light AR, Veranth JM, and Reilly CA (2013) Activation of transient receptor potential ankyrin-1 (TRPA1) in lung cells by wood smoke particulate material. Chem Res Toxicol 26:750-758 Available from: 10.1021/tx400024h.

Sukumaran P, Sun Y, Zangbede FQ, da Conceicao VN, Mishra B, and Singh BB (2019) TRPC1 expression and function inhibit ER stress and cell death in salivary gland cells. FASEB Bioadv 1:40-50.

Swiston JR, Davidson W, Attridge S, Li GT, Brauer M, and van Eeden SF (2008) Wood smoke exposure induces a pulmonary and systemic inflammatory response in firefighters. Eur Respir $J$ 32:129-138.

Thomas KC, Ethirajan M, Shahrokh K, Sun H, Lee J, Cheatham TE III, Yost GS, and Reilly CA (2011) Structure-activity relationship of capsaicin analogs and transient receptor potential vanilloid 1-mediated human lung epithelial cell toxicity. J Pharmacol Exp Ther 337:400-410.

Thomas KC, Roberts JK, Deering-Rice CE, Romero EG, Dull RO, Lee J, Yost GS, and Reilly CA (2012) Contributions of TRPV1, endovanilloids, and endoplasmic reticulum stress in lung cell death in vitro and lung injury. Am J Physiol Lung Cell Mol Physiol 302:L111-L119.

Thomas KC, Sabnis AS, Johansen ME, Lanza DL, Moos PJ, Yost GS, and Reilly CA (2007) Transient receptor potential vanilloid 1 agonists cause endoplasmic reticulum stress and cell death in human lung cells. J Pharmacol Exp Ther 321: 830-838.

Wang Y, Wu Z-Z, and Wang W (2017) Inhibition of endoplasmic reticulum stress alleviates cigarette smoke-induced airway inflammation and emphysema. Oncotarget 8:77685-77695.

Watterson TL, Hamilton B, Martin R, and Coulombe RA Jr. (2009) Urban particulate matter causes ER stress and the unfolded protein response in human lung cells. Toxicol Sci 112:111-122.

Xu C, Bailly-Maitre B, and Reed JC (2005) Endoplasmic reticulum stress: cell life and death decisions. $J$ Clin Invest 115:2656-2664.

Xu C, Luo J, He L, Montell C, and Perrimon N (2017) Oxidative stress induces stem cell proliferation via TRPA1/RyR-mediated $\mathrm{Ca}^{2+}$ signaling in the Drosophila midgut. eLife 6:e22441.

Xu M, Zhang Y, Wang M, Zhang H, Chen Y, Adcock IM, Chung KF, Mo J, Zhang Y, and Li F (2019) TRPV1 and TRPA1 in lung inflammation and airway hyperresponsiveness induced by fine particulate matter $\left(\mathrm{PM}_{2.5}\right)$. Oxid Med Cell Longev 2019:7450151.

Yao Y, Wang Y, Zhang Z, He L, Zhu J, Zhang M, He X, Cheng Z, Ao Q, Cao Y, et al. (2016) Chop deficiency protects mice against bleomycin-induced pulmonary fibrosis by attenuating M2 macrophage production. Mol Ther 24:915-925.

Address correspondence to: Dr. Christopher A. Reilly, Department of Pharmacology and Toxicology, University of Utah, 30 South 2000 East, 201 Skaggs Hall, Salt Lake City, UT 84112. E-mail: Chris.Reilly@pharm.utah.edu 